\title{
I Mostri, i nuovi Mostri e. . . . ricomincio da tre: l'évolution de la comédie dans le cinéma italien.*
}

En ce début des années quatre-vingts, il y a lieu de se demander s'il est possible de parler de nouvelle comédie italienne. Y a-t-il assez de constantes, de points communs entre les nouveaux auteurs comiques pour que l'on puisse d'ores et déjà esquisser une définition de la nouvelle comédie italienne? Avant d'aborder la question, il conviendrait de faire un retour en arrière pour délimiter les contours de la comédie italienne; reconsidérer brièvement sa place au sein de l'histoire du cinéma italien, et surtout essayer de comprendre pourquoi la comédie, longtemps perçue comme un genre mineur, a été boycottée pendant de nombreuses années par la critique, surtout en Italie.

A la racine de cette indifférence, voire de ce mépris, ${ }^{1}$ il semble y avoir un préjugé d'ordre intellectuel qui amène une certaine critique à percevoir le film idéal comme une oeuvre confidentielle s'adressant à une élite. Confondre le succès populaire de la comédie avec un vil succès commercial en quête de pittoresque signifie ne pas saisir l'essence de ce genre artistique. Il a toujours existé une attitude ambivalente face au rire: est-il impossible de se distraire en traitant de sujets graves? C'est justement ce refus du mélange du rire et des larmes qui a fait poser des jugements inexacts et quelque peu simplistes sur la comédie italienne. D'un côté, on lui a reproché son dilettantisme et sa superficialité, de l'autre une prédilection pour des thèmes vulgaires aux odeurs de latrine et de bordel. Pourtant, il suffit de relire les oeuvres théâtrales de Plaute ou encore mieux La Mandragola de Machiavelli, pour retrouver les origines de ce comique grotesque et plébéen. Bien sûr, il y a façon et façon de faire rire, mais "la comédie est par nature sceptique, moqueuse, pragmatique et elle s'attache aux aspects quotidiens voire triviaux de la vie." ${ }^{2} \mathrm{Or}$, la comédie dans le cinéma italien comme dans le théâtre comique traditionnel, se sert du vulgaire comme moyen corrosif pour souligner le gro- 
tesque au bord de l'autodestruction. Quant à l'accusation de "macchiettismo" portée contre la comédie italienne, on peut y répondre en citant ces propos de Lattuada: "Même L'Enfer de Dante est une sorte de portrait de famille, une série de petits portraits, de nouvelles replacées dans un contexte universel. ${ }^{3}$ La comédie accède à l'universalité par le biais du quotidien et par son refus de se laisser embrigader dans une idéologie rigoureuse.

Ce qu'il y a de paradoxal à propos de la comédie dans le cinéma italien c'est que, à partir du moment où elle a commencé à être reconnue voire enseignée dans les universités, elle a manifesté quelques signes de fatigue. En outre, sa reconnaissance à l'étranger surtout en France à partir des années soixante-dix -, plutôt que de l'aider, semble l'avoir incitée à se reposer sur ses lauriers. Ce qui a été défini comme "la comédie à l'italienne" est maintenant en crise et ne semble plus en mesure de renouveler ses fondements et ses thèmes. L'hommage rendu en France à l'originalité et à la vitalité de ce genre, a donc pris le visage d'une célébration post mortem. Certes, les éléments thématiques et esthétiques qui ont caracterisé la comédie pendant les années soixante et soixante-dix, ont perdu leur cohésion et leur spécificité à cause de la difficulté rencontrée par les cinéastes de trouver à l'heure actuelle des cibles facilement identifiables et attaquables. Cependant, la comédie manifeste encore aujourd'hui sa présence dans le cinéma italien de deux façons différentes. D'un côté, le grotesque et la satire sociale que l'on retrouve dans les films de Marco Ferreri ou même de Federico Fellini s'apparentent beaucoup à des comédies désormais classiques comme I Mostri de Risi par exemple. De l'autre, l'essoufflement de toute une génération de metteurs en scène comiques dans la cinquantaine a permis l'éclosion d'une réinterprétation de la comédie, favorisant l'intégration d'éléments burlesques à la Keaton dans Ratataplan de Nichetti ou bien un retour à la gestualité de la commedia dell'arte comme dans Ricomincio da tre de Troisi.

\section{L'influence française}

La comédie s'est constituée par étapes avant que ses caractéristiques ne se stabilisent. Suivons le filon historique qui semble apte à nous conduire diachroniquement à une compréhension synchronique de la comédie.

A l'époque muet, c'est-à-dire au cours des années dix, le cinéma italien ne manquait pas de talents comiques mais, étrangement, les plus fameux d'entre eux étaient d'origine étrangère. En effet, les maisons de production italiennes d'alors engageaient 
surtout des acteurs comiques français dont l'un d'entre eux, André Deed, connu en France sous le nom de Boireau, deviendra célèbre sous le pseudonyme de Cretinetti et fera la fortune de la société Itala établie à Turin. Lui, ainsi que Marcel Fabre alias Robinetti, Natale Guillaume alias Cocciatelli et Fernandino Guillaume alias Tontolini puis Polidor, seront les grandes vedettes d'un comique destructeur, cousin du "slapstick" qu'il préfigure. Parallèlement à ce dernier, se développe à travers les "commedie familiari" un comique vaudevillesque. Il convient de souligner que dès cette époque, les metteurs en scène italiens, mieux que leurs cousins français, savaient soigner leurs décors et leurs costumes, varier leurs cadrages, rythmer les mouvements de leurs personnages à l'intérieur du champs, pour s'éloigner de la banale reproduction filmique d'un spectacle de théâtre. Même dans une situation comique, les personnages oeuvraient parmi des décors réalistes. Nous décelons là une imitation de la reconstitution historique qui caractérisait une grande partie du cinéma italien de cette époque et dont, par exemple, Enrico Guazzoni avec un film tel que Quo Vadis a été l'un des plus illustres représentants, sans oublier Pastrone avec le désormais classique Cabiria. Toutefois, l'on peut dire que l'époque du muet comique a été plus l'époque du burlesque que du vaudevillesque. Paradoxalement, le burlesque n'a jamais véritablement trouvé sa terre d'élection en Italie, ce qui explique en partie le recours fréquent aux acteurs étrangers que l'on avait à l'époque.

\section{Les "Dive," "I telefoni bianchi" et Totò}

Le phénomène des "dive" qui obligeait les réalisateurs à travailler en fonction des caprices et dégoûts de leurs vedettes féminines est une des causes principales de la crise qui frappe le cinéma italien pendant la première guerre mondiale. Durant les années vingts, l'arrivée du fascisme au pouvoir ne change guère la situation, même si Mussolini, en 1934, par le biais du Ministère de la Culture populaire, crée la Direction Générale de la Cinématographie, en 1935, "l'Istituto Luce" et le "Centro sperimentale," puis en 1937 les studios de "Cinecittà" afin d'augmenter la production filmique. Le spectateur d'alors voit défiler sous ses yeux une suite de films fabriqués sur commande, sans spécificité régionale et sans originalité. C'est à ce moment-là qu'apparaissent les comédies bourgeoises dites de "telefoni bianchi" qui exaltent dans la facticité et la superficialité de leurs décors, un sentimentalisme aussi mièvre que mondain, un romanesque-évasion où le préfabriqué joue un rôle compensatoire et mystifiant, ce qui s'inscrit 
dans la politique du régime. L'on y sent l'influence prépondérante de la "screwball comedy," plus souvent qu'à son tour laminée, débarrassée de son contenu pour n'être plus qu'une apparence d'elle-même, en dépit de son élégance de style. On a coutume de signaler dans ce vaste désert de l'histoire du cinéma fasciste quelques oasis se distinguant de la médiocrité générale: les metteurs en scène Matarazzo, Blasetti et Camerini et les acteurs Petrolini et Viviani. Avec "sa comicité impitoyable", pour reprendre l'expression de Filippo Sacchi, le grand acteur Ettore Petrolini devient le chef de file d'un comique populaire puisant son inspiration dans l'atmosphère de l'époque, mais aussi le premier représentant d'un comique cinématographique qui hérite d'un répertoire et d'un style élaborés sur les scènes de "l'avanspettacolo." Dans les jeux de Petrolini et Viviani, l'on perçoit les premiers fondements de la comédie italienne, c'est-à-dire un mélange de grotesque et de notations subtiles où le comique embrasse le désespoir. En 1933, avec Treno popolare, Raffaele Matarazzo, par le regard à la fois ironique et critique qu'il pose sur son sujet, par l'aspect documentaire et la pauvreté des moyens de son film, par l'utilisation d'acteurs non-célèbres, s'affirme comme l'un des plus lointains précurseurs du néo-réalisme. Dans La Tavola dei Poveri, Alessandro Blasetti, en mettant en scène contre toute rhétorique officielle la misère méridionale, fait discrètement poindre le drame sous l'humour, drame de la solitude, du chômage et de la pauvreté qui sera le thème sacré du cinéma néo-réaliste, avant de s'affirmer comme l'un des ressorts de la comédie italienne. A la même époque, Mario Camerini avec Gli Uomini, che Mascalzoni, très proche par le ton de Sous les toits de Paris ou Quatorze juillet, construit une histoire d'amour simple qui refuse de sacrifier à la vogue des personnages hors de commun comme Scipione l'Africano, exaltés par le régime fasciste. Les comédies brillantes et quelque peu sophistiquées de Mario Camerini, en pervertissant les conventions qu'elles sont censées servir, en écartant jusqu'à un certain point le vide des comédies de "telefoni bianchi" par l'ajout d'un substrat social, nous semblent elles aussi ouvrir la voie au cinéma néo-réaliste qui à son tour va influencer la comédie italienne des années cinquante. L'importance de Mario Camerini dans l'histoire du cinéma italien est encore plus perceptible à travers sa collaboration avec les célèbres frères napolitains, Eduardo et Peppino De Filippo à qui il donnera leur premier rôle cinématographique dans Il Cappello a tre punte. Par l'entremise de Eduardo De Filippo dont on filme les pièces, le théâtre dialectal et la tradition mimique napolitaine, issus de la commedia dell'arte, font leur entrée dans le cinéma comique italien. Grâce au théâtre dia- 
lectal, la comédie italienne revient à ses racines profondes. C'est l'art de "la pernacchia" pouvant être défini comme la libération par l'absurde ou une folie joyeuse triviale, qui fait un retour en force. Comme le précise Garel dans son article "la comédie italienne et la critique," ${ }^{5}$ "le comique, même s'il prend sa source dans la comédie gréco-latine, emprunte à la verve populaire ses détails grotesques, ses costumes, ses jeux de mots et ses allusions grossières et scabreuses." La commedia dell'arte inspirera les plus grands acteurs comiques italiens, que ce soit le génial Totò, le tandem Franco Franchi et Ciccio Ingrassia ou les Monstres, c'està-dire Sordi, Gassman, Tognazzi et Manfredi. Avec Antonio De Curtis alias Totò, se perpetue la tradition du tragique napolitain aux couleurs de la farce grotesque. Il est, nous dit Benayoun, "le Pulcinella de la tradition classique ... Il a fait de son physique lunaire un usage résolument agressif: sa triste mine de valet enfariné peut, d'un seul coup, se strier d'un sourire avantageux, assassin. Et pourtant Totò n'a jamais eu de prix et Totò est un génie." $"$

\section{Comédie et néo-réalisme}

Comme nous l'avons déjà précisé, la comédie populaire fait partie des origines du néo-réalisme, d'une part parce que les cinéastes fatigués des comédies bourgeoises dénuées de tout message social ont décidé de descendre dans la rue, d'autre part, parce qu'en contre-réaction à la volonté nationale d'uniformisation de Mussolini, il y a eu un retour en force aux langages dialectaux. Pour confirmer cette hypothèse, il suffit de citer les noms de Anna Magnani ou Aldo Fabrizi qui ont débuté comme acteurs comiques avant de se lancer dans des rôles dramatiques comme ceux de Roma città aperta, pour reprendre quatre ans plus tard leurs personnages de poissarde et de maraîcher dans L'Onorevole Angelina. En 1942-43, Fabrizi en collaborant avec Fellini et Zavattini aux scénarios de Avanti, c'è posto, de Campo de' fiori et de L'ultima carrozzella, devient le créateur d'un univers particulier qui s'affirme comme un véritable exorde du néo-réalisme. Par une sorte de phénomène de boomerang, le cinéma néo-réaliste influencera à son tour la comédie en lui faisant prendre conscience des problèmes de la société italienne. On a accusé à tort la comédie italienne d'avoir adultéré les éléments néo-réalistes à des fins pittoresques. ${ }^{7}$ Il est indéniable que le néo-réalisme a donné un fondement social à la comédie italienne. Mais ceci n'est pas son seul apport: en effet, au moment de son déclin, vers le milieu des années cinquante, un certain nombre de cinéastes s'est regroupé 
en adoptant le comique comme Koine filmique. Même un des chefs de file du néo-réalisme, Vittorio De Sica, fait un retour en tant qu'interprète à des comédies de type Pane, amore e fantasia. Il faut souligner en outre que la comédie italienne des années cinquante incorpore le style quasi-documentaire du néo-réalisme, ce qui la différencie profondément de la comédie des années trente.

\section{Du Marco Aurelio à la satire sociale}

L'hebdomadaire satirique romain, Marco Aurelio, a été le lieu de rencontre d'un groupe de jeunes journalistes comme Steno, Monicelli, Age, Scarpelli, Scola et Maccari qui deviennent ensuite scénaristes de comédies. Cette expérience commune favorise leur cohésion intellectuelle qui se manifeste par une écriture où la plume vive de la peinture satirique se greffe au fond austère de la description réaliste. Même Antonioni, réputé pour son réalisme dépouillé, collabore à l'élaboration d'un scénario comique comme celui du "Sceicco bianco." Pendant deux décennies donc, les échanges entre scénaristes dont certains comme Scola deviendront cinéastes, ne cessent pas et le travail collectif fait converger régulièrement leurs trajectoires individuelles. Par conséquent, le film è sketches, canevas fondamental de la comédie italienne souvent construite selon une technique rhapsodique, est une des manifestations de cet esprit "collégial," de ce travail en commun. Par exemple, le film Signore et signori, buona sera est le produit d'une coopérative fondée par des cinéastes, des écrivains et des comédiens.

Les années cinquante voient donc émerger un groupe de metteurs en scène ayant pour nom Germi, Monicelli, Risi, Comencini, Lattuada, tous issus de familles aisées, de milieux cultivés et originaires du nord. Ces cinéastes nordiques utilisent un humour corrosif pour exprimer leur refoulement et leur indignation face au "qualunquismo" et aux vices nationaux, ce qui leur permet d'atteindre une profonde osmose avec le public populaire qui se reconnaît dans leurs films. Ces metteurs en scène s'attachent à décrire principalement les avatars du prolétariat. C'est seulement à partir des années soixante-dix qu'une thématique proprement bourgeoise apparaît dans leurs oeuvres. Mais l'angle de vision et le but visé demeurent malgré tout populistes, ce qui fait dire à Miccichè que "la comédie est toujours restée qualunquiste. ${ }^{8} \mathrm{Ce}$ populisme frise la condescendance parce qu'il brosse le plus souvent le portrait caricatural de l'Italien méridional capté dans son décor naturel. Cependant, il faut remarquer qu'à partir des années soixante-dix, le type de situations auxquels les cinéastes s'in- 
téressent, les obligent à tourner sur tout le territoire national. Ainsi, Monicelli transpose Amici miei à Florence, sa ville natale, alors que le Milanais Risi fait un retour à la Lombardie dans $\mathrm{La}$ Stanza del Vescovo, Primo Amore et Fantasma d'amore. D'ailleurs, Risi a toujours montré dans ses oeuvres, le goût du voyage, de la découverte du pays, voire de l'itinéraire picaresque: de Turin à Naples dans Profumo di donna, de Milan à Rome dans Una vita difficile, de Rome à la Toscane dans Il Sorpasso.

\section{Les monstres}

Comme les metteurs en scène pour lesquels ils travaillent, les grands acteurs comiques italiens que l'on a surnommé à juste titre "les Monstres," forment un clan et ont de nombreux points communs. Manfredi et Sordi viennent du sud alors que Tognazzi et Gassman sont originaires du nord. Avant de s'adonner au cinéma, Manfredi, Sordi et Tognazzi ont oeuvré dans le cabaret, le music-hall ou dans les "avanspettacoli." Vittorio Gassman, lui, a débuté au théâtre classique où il s'est distingué dans de grands rôles tragiques. Tous les quatre ont également fait l'expérience de la mise en scène. ${ }^{9}$ Si le public a toujours aimé ces quatre grands monstres - nous ne parlerons pas ici de Mastroianni dont le cas est un peu à part car il fait de nombreux rôles de composition aussi bien comiques que dramatiques —, c'est parce qu'il s'identifie jusqu'à un certain point à ceux-ci et qu'il retrouve en eux les multiples prototypes de l'Italien moyen. En fait, Sordi, Manfredi, Tognazzi et Gassman, non seulement sont les interprètes des meilleures comédies, mais ils sont également des masques caricaturaux, des portraits-types, qui, sous des dehors anodins, révèlent par le biais du grotesque la monstruosité humaine. Sordi, profondément romain, incarne toujours une sorte de héros négatif, à la fois poltron, pessimiste, résigné, qui pratique "l'arte di arrangiarsi" et qui, à l'image du "borghese piccolo, piccolo" de Monicelli, révèle sous des dehors aimables, une immaturité dangereuse et agressive. Manfredi, contrairement à Sordi, s'éloigne de tout grotesque et représente avec douceur et une mélancolie aux résonnances chaplinesques, le sympathique et bavard "ciociaro," victime en puissance, qui, comme les héros de Pane e Cioccolata ou Caffè espresso s'en sort toujours par son intuition et sa richesse intérieure. Tognazzi figure l'Italien dans ses aspects les plus viscéraux. Epicurien, c'est un héros de "grande bouffe," toujours en parfaite harmonie avec les forces de la Nature. Gassman, parmi ces quatre acteurs, est celui qui dégage le plus de prestance. Il personnifie le matamore, l'Italien qui sous des dehors 
braves, reste profondément froussard. Comme Santenocito dans Nel nome del popolo italiano, il incarne le type d'homme bien nanti, cultivé, distingué, qui cache son désarroi intérieur par une multitude d'occupations et un verbiage incessant.

Les actrices jouent-elles un rôle semblable à celui des vedettes masculines dans les comédies? Il nous semble que non: évidemment, il y a des comédiennes comme Monica Vitti et Mariangela Melato qui, pendant les années soixante et soixante-dix, ont pris la relève de Sophia Loren et se sont livrées à une parodie de différents types féminins. Toutefois, la femme reste en arrière-plan ou constitue un aspect complémentaire de l'homme dans la plupart des comédies italiennes dont émane l'image d'un patriarcat encore profondément enraciné dans la réalité sociale. Si on regarde l'ensemble des rôles joués par les femmes dans les comédies, on ne réussit pas à saisir leur portrait-robot avec acuité comme on perçoit, au contraire, celui de l'homme italien moyen. Dans les films de Lina Wertmuller, la présence féminine est certes importante, mais elle est toujours analysée en fonction d'un rapport dominant-dominé. L'exemple le plus probant en est cette sorte de parabole sociale ayant pour titre: Travolti da un insolito destino nell'azzurro mare d'agosto. Monica Vitti aussi joue un rôle semblable à celui de Mariangela Melato dans Dramma della gelosia où elle reste en fin de compte l'objet convoité de deux amants. Les femmes donc ne sont pas des "Monstres" car leur rôle n'acquiert son sens qu'à travers celui de l'homme. Selon nous, les cinéastes comiques italiens - même Wertmuller —, tout en étant fascinés par la femme ne saisissent pas sa complexité. La plupart du temps, ils la perçoivent comme une créature de romans-photos - les nombreux rôles joués par la Belli, la Muti, la Antonelli ou la Sandrelli corroborent cette image - , ou bien comme la femme-mère ou "mamma" qu'on retrouve même dans un film aussi récent que $R i$ comincio da tre de Troisi.

Le pilier de la comédie, en fin de compte, reste l'acteur, l'actrice jouant un rôle ancillaire par rapport à lui. C'est à travers lui que le public réagit, est impliqué dans le double jeu de la mimesis et de la distanciation et participe à la peinture globale des moeurs de son pays. "Comme les toiles d'Arcimboldo où le visage d'un homme est un puzzle complexe de fruits variés, le portrait composite de l'Italien moyen pourrait s'obtenir par la surimpression des cing comédiens qui dominent le cinéma italien d'aprèsguerre." ${ }^{10}$ Ces Montres, version moderne des masques de la commedia dell'arte, ont concrétisé à maintes reprises, les faiblesses, les travers, les fantasmes du peuple pour tenter de répondre à cette éternelle question: pourquoi sommes-nous comme nous sommes? 
Ce sont justement les tentatives de répondre à cette question qui donnent à la comédie sa pleine signification, en plus de lui avoir permis de devenir une véritable satire des moeurs. Ainsi, les vices nationaux ont été illustrés plus par la satire que par toute autre forme d'expression artistique, en particulier le cinéma engagé. En effet, contrairement à celui-ci, la comédie, malgré sa sympathie pour la classe ouvrière, ose montrer les pauvres même dans leur bassesse. L'ambiguïté de l'âme humaine est mieux perçue par le rire grinçant que par le sérieux du cinéma engagé dont le manichéisme est au service d'une thèse à démontrer. Cette affirmation nous permet de contester directement les propos d'un critique comme Lino Miccichè qui voit dans la comédie italienne une représentation d'une vision "qualunquiste" de la société et perçoit dans le discours que la critique française tient à propos de ce genre cinématographique, un colonialisme en mal d'exotisme. Il en arrive même à dire qu'en France, l'amour de la comédie italienne est le reflet d'un snobisme culturel, d'une certaine inclination pour "le petit nègre" transalpin. Mais, selon nous, si les Français l'aiment tellement, c'est qu'ils retrouvent en elle un véritable miroir déformant de la réalité sociale et culturelle d'un pays, alors que les comédies pâlotes de l'Hexagone où De Funès prend la vedette, correspondent è une suite de gags sans aucune incidence sur le réel.

\section{Du boom au "Compromesso Storico"}

Au début des années soixante, à cause de l'industrialisation rapide du pays et de ce que Pasolini appelait "la révolution anthropologique de l'Italien," la problématique sociale s'enrichit. La comédie devient donc l'outil caricatural qui souligne le décalage entre l'évolution sociale en cours et le refus d'abandonner certains comportements typiques d'une société agricole, tels que la fidélité conjugale, le sens de l'honneur, auquel se rattache le mythe sacré de la virginité. Après I soliti Ignoti (1958) considéré comme le précurseur d'une comédie plus humoristique et plus soignée dans sa forme, les metteurs en scène comiques réalisent une série d'oeuvres qui brossent un tableau des thèmes nouveaux engendrés par l'évolution historique du pays. ${ }^{11}$

Vers la fin des années soixante-dix, la comédie est en perte de vitesse. Dans un contexte social où les valeurs antynomiques se confondent, les scénaristes ont de plus en plus de difficultés à s'en prendre à d'aléatoires institutions. En ce sens, I nuovi Mostri, paradigme de la comédie italienne, résume à lui seul la monstrueuse réalité d'aujourd'hui qui dépasse les fictions les plus 
folles. On peut faire coïncider la crise du cinéma italien avec l'atmosphère sociale engendrée par le "compromesso storico" de 1976. Pendant six ans - c'est-à-dire jusqu'à la formation du gouvernement Spadolini en 1981 - il n'y aura plus en Italie de différence précise entre l'opposition et la Démocratie Chrétienne. La politique italienne connaîtra une impasse, et même la gauche ne sera pas exempte de corruption. Or, les cinéastes comiques italiens, à l'exception de Scola, même s'ils ne s'affirmaient pas de gauche, trouvaient auparavant dans la Démocratie Chrétienne et le système de valeurs qui s'y rattachait, une cible fixe qu'ils aimaient à fustiger. Mais les metteurs en scène comme les acteursmonstres ont vieilli et ils ont de la difficulté à évoluer autant sur le plan professionnel qu'idéologique. Le film de Scola, La Terrazza, exprime très bien l'impasse dans laquelle se trouve une génération de cinéastes de gauche pour qui l'appartenance idéologique avait un rôle historique précis à jouer. L'ironie leur sert dès lors de bouclier pour faire le constat de leurs échecs, pour survivre à leurs erreurs tout en s'avouant leur désarroi.

\section{De Celentano à la relève des années quatre-vingts}

Il est indéniable qu'à l'heure actuelle, les auteurs comiques traditionnels italiens sont essouflés. Ils essaient malgré tout d'évoluer vers de nouvelles directions où, comme dans Fantasma d'amore (1981) de Risi et Il Matrimonio di Caterina (1982) de Comencini, le grotesque de la comédie italienne s'efface pour laisser place au lyrisme romantique. En même temps, une foule de productions de troisième ordre dont l'humour ne dépasse guère les limites de vaudeville, du music-hall, et dont la portée critique est inexistante, continue d'envahir les écrans de la Péninsule. Cette vague de films "farcesques" a vu naître avec elle une nouvelle génération d'interprètes comiques polyvalents, pour la plupart issus des milieux de la chanson ou de la télévision, tels que les comédienschanteurs Johnny Dorelli, Adriano Celentano, Massimo Ranieri ou les artistes de cabaret Cochi Ponzoni, Renato Pozzetto, Enrico Montesano et Roberto Benigni. Un bon nombre de films dans lesquels ces artistes jouent sont construits de façon à mettre en évidence leur talent de chanteurs ou de meneurs de shows. Voilà pourquoi ils ne sont pas en mesure d'accéder au statut de "monstre sacré." Le plus prometteur de cette nouvelle génération d'interprètes comiques semble être Roberto Benigni, qui, après avoir été dirigé en 1977 par Giuseppe Bertolucci dans Berlinguer, ti voglio bene, a révélé son potentiel dans Chiedo asilo (1979) de Marco Ferreri. Quant au tandem Melato-Giannini, principaux protagonistes 
des films de Lina Wertmuller, il s'est désagrégé au fil des années puisqu'il refuse désormais de se confiner au seul genre comique.

\section{"Ricomincio da tre" et la nouvelle comédie}

En ce début des années quatre-vingts, la véritable relève sur laquelle se fondent les espoirs d'une nouvelle comédie, se résume à quatre noms: Maurizio Nichetti, Nanni Moretti, Carlo Vedone et Massimo Troisi. C'est ce dernier qui nous intéresse tout particulièrement car il nous semble être la grande révélation du cinéma comique italien d'ajourd'hui. ${ }^{12}$ L'originalité de ce jeune cinéaste s'est manifestée dans son "opera prima": Ricomincio da tre. Le film raconte une histoire très simple: la relation amoureuse d'un jeune Napolitain timide, Gaetano, avec une jolie Florentine délurée, Marta. Au-delà de cette charmante romance, ce sont les nouveaux rapports entre l'homme et la femme, également représentatifs de la problématique traditionnelle nord-sud, qui sont analysés. Si Gaetano décide de quitter Naples pour aller à Florence, ce n'est pas, contrairement à ce qu'on lui rappelle continuellement, pour émigrer ou aller $\mathrm{y}$ chercher du travail, mais simplement pour changer d'air et vivre de nouvelles expériences. Pour Gaetano, son voyage est un prétexte à une étude fine et satirique des comportements à la mode, comme un goût prononcé pour l'exotique et le bizarre ou un snobisme intellectuel nombriliste propre à une classe aisée. C'est ainsi que Troisi manifeste son originalité et se différencie de ses prédécesseurs septentrionaux, trop enclins à se pencher avec condescendance sur les pauvres gens du sud pour des raisons idéologiques ou par une espèce de provincialisme nostalgique à la Pasolini. Ici, les rôles sont inversés et la problématique nord-sud est d'autant plus intéressante qu'elle est abordée d'une manière inusuelle. La démarche de Troisi s'intègre à une tentative intellectualiste caractéristique du nouveau cinéma comique italien, soucieux de dénoncer les récents totems et tabous d'une bourgeoisie moyenne qui s'adonne à la méditation, à la parapsychologie, à la psychanalyse, aux herbes et aux restaurants macrobiotiques. Par le biais des situations drôlatiques du quotidien et par son regard détaché et faussement naï, Troisi saisit les comportements sociaux dans toute leur complexité et il accède à une profonde réflexion sur le sens de la vie et du sacré. Pour y arriver, il invente une forme très originale de monologue basée sur l'accumulation verbale, monologue qui décape les valeurs traditionelles du sud et nouvelles du nord. Alors que Marta est toujours présentée seule, libérée, sans attaches familiales, avec un emploi et de jeunes amis issus du même milieu qu'elle, Gaetano, lui, ap- 
paraît toujours dans un contexte où les valeurs traditionnelles, comme les attaches familiales, étouffent son individualité.

La vision de Troisi se différencie de celle de Nanni Moretti. En effet, ce dernier démystifie par la parodie la contre-culture issue de mai soixante-huit. L'oeuvre de Moretti, nous semble-t-il, comporte des limites certaines. En effet, le cinéaste se caricature luimême puisqu'il appartient à l'intelligentsia romaine de gauche qui fait l'enjeu de sa critique. Il manifeste ainsi un certain narcissisme dans sa démarche, laquelle ne dépasse pas les limites de son groupuscule. Il se dégage de son oeuvre - les titres de ses films: Sono un autarchico, Ecco Bombo et Sogni d'oro sont là pour le prouver - une sorte d'humour potache et égocentrique peu ancré dans la réalité italienne et difficilement accessible à un public non-averti. Maurizio Nichetti, lui, s'attache à décrire les problèmes d'une jeunesse à son image, intellectuelle, artiste, paumée dans le labyrinthe technocratique de Milan, avec un enthousiasme et un vitalisme aux limites de l'idéalisme ingénu: dans son dernier film Domani si balla, une étrange épidémie de la danse, en provenance d'un monde inconnu, porte à nouveau la joie sur terre et efface toutes les peines des hommes! Une telle conception du monde risque de faire tourner en rond le jeune auteur dont "l'opera prima," Ratataplan, était pourtant prometteuse. A moins d'avoir le génie de Chaplin et de Keaton, il y a un danger à reprendre d'un film à l'autre exactement le même personnage. Le cas de Moretti et de Nichetti qui ne savent jouer rien d'autre qu'eux-mêmes laisse quelques doutes sur leur avenir de comédien.

Si le héros incarné par Moretti est une sorte de "Candide" désopilant et un peu mélancolique qui se promène dans les sous-bois culturels de Rome, et dont les gros plans muets dégagent un comique à cheval entre Lewis et Keaton, celui de Nichetti, avec son pantalon trop court, ses moustaches au vent et son badge à l'envers, rappelle à la fois Zani, l'antique masque du paysan naif et un peu simple de la commedia dell'arte, et l'Auguste - celui qui forme avec le clown blanc le duo classique du cirque. Le personnage de Nichetti ressent très nettement l'apport du cinéma muet américain, ce qui nous laisse croire qu'il aspire à être le neveu latin de Keaton et de Chaplin. Chez ce cinéaste, le comique revêt principalement les formes du burlesque américain, de la tradition du cirque tout en faisant référence à l'univers comique de Tati, alors que chez Moretti, il se renouvelle surtout par la parodie de café-théâtre qui n'est pas sans rappeler celle des "Frustrés" de Bretecher.

Tandis que ses confrères s'inspirent de modèles étrangers qu'ils tâchent d'italianiser, Troisi, dont le respect de la tradition indi- 
gène est plus fort en raison de son ascendance méridionale, fait de son personnage un Pulcinella en costume de ville avec des gestes et un humour verbal directement inspirés de la tradition mimique napolitaine. Chez lui, le discours accompagné de gestes précis est le ressort principal du comique qui trouve réponse à tout. Ainsi, la migration des oiseaux est due, selon Gaetano, à Saint-François qui les dérangeait en voulant converser avec eux! La portée épistémologique de la méthode employée par Troisi couvre un champ très vaste. Il veut atteindre les grandes vérités par une série de mots d'esprit. Chez Moretti et Nichetti, la recherche de la signification a, au contraire, une portée plus restreinte. Le premier base en grande partie son comique sur les répétitions tautologiques qui vident le discours de tout sens, alors que le second, dans ses films, exclut presque entièrement le dialogue au profit de l'image. ${ }^{13}$

Malgré les différences que nous venons de mentionner, pour tous ces jeunes auteurs comiques, le cinéma est d'abord une affaire de famille où chacun, à l'intérieur d'une structure professionnelle et industrielle, essaie de créer un climat amical. Aux grandes vedettes, ils préfèrent leurs amis journalistes, professeurs, étudiants et musiciens avec lesquels, à partir d'un canevas souple, ils improvisent au moment du tournage. Cette improvisation favorise l'élaboration d'un style paratactique fait d'une suite de petits tableaux. Une telle façon de procéder, à laquelle vient s'ajouter la modestie des moyens mis en oeuvre, a ses bons et ses mauvais côtés. Elle occasionne certaines faiblesses au niveau de la cohérence du récit qui donne une impression de décousu et n'est pas exempt de certaines longueurs, ainsi qu'au niveau de la technique dont on se préoccupe peu ou pas. Nichetti filme mal et fait un emploi exagéré du zoom, Moretti abuse des plans fixes et bavards, et ses images sont négligées à force d'être simples; quant à Troisi, il se débrouille mieux que ses camarades, même si sa caméra demeure quelque peu passive dans son désir de remplir l'espace par le geste. D'autre part, ce retour à une simplification des formes qui contraste avec une intellectualisation des thèmes sexe, violence et vulgarité sont soigneusement exclus - confèrent aux nouvelles comédies italiennes une authenticité qui les éloigne de la calligraphie et de la titillation de la nudité de la femme, si présente dans les sous-produits cinématographiques.

Est-il possible de donner une définition claire de la nouvelle comédie italienne? Les films des jeunes réalisateurs comiques possèdent-ils assez de traits communs pour parler de renouveau d'un genre? Il est indéniable selon nous que chez de jeunes cinéastes comme Moretti, Nichetti et Troisi, existe une nouvelle conception 
du comique et qu'il y a chez eux une volonté de rupture esthétique par rapport à la comédie des années soixante. En comparant un de leurs films avec une oeuvre comme Il Sorpasso de Risi ou Nudo di donna de Manfredi (un des quatre monstres devenu réalisateur), l'on perçoit une prédilection pour des thèmes différents, tels que la jeunesse, le snobisme factice de "l'esterofilia," la libération apparente des moeurs sexuelles ou le cynisme face aux absolus de l'idéologie. Parallèlement, il y a un désir de traiter les formes du récit cinématographique d'une manière nouvelle: l'élément qui nous semble le mieux la caractériser est la substitution du grotesque par le burlesque, ce qui réduit la portée mimétique de la comédie et souligne au contraire le côté mimique des comportements. De toutes les oeuvres de ces jeunes réalisateurs, "l'opera prima" de Troisi, Ricomincio da tre, nous apparaît comme l'exemple le plus prometteur d'un renouvellement du contenu et des formes de la comédie. Troisi réussit à atteindre un comique qui repose sur les jeux visuels constamment corroborés par des calembours. En outre, la vision sociale de Troisi est très étendue puisque la dialectique nord-sud est présente, et analysée à partir d'une sensibilité méridionale qui élimine les risques de la condescendance gratuite.

Massimo Troisi vient de terminer son deuxième film, Scusate il ritardo, racontant l'histoire de Vincenzo, un jeune Napolitain acâriatre et rationnel, aux prises avec ses problèmes d'amour. Cette trame ressemble étrangement à celle de Ricomincio da tre. Devonsnous conclure que même chez Troisi le risque d'un narcissisme qui apparaît déjà de manière évidente dans les oeuvres de $\mathrm{Ni}$ chetti et Moretti, est inévitable? Mais Pulcinella, rappelons-le, possède une vitalité inépuisable. ...

\section{Concordia University}

\section{NOTES}

* Cet article est la version remaniée d'une conférence donnée par Anna Gural le 28/04/83 à la Cinémathèque québécoise.

1 Exception faite pour les Français Bazin, Chapier, Moullet et Siclier, - collaborateurs aux Cahiers du Cinéma —, et pour les Italiens Camolli et Chiarini.

2 Michel Ciment, "La comédie italienne," in L'Express magazine (sept. 78), 18.

3 "La comédie italienne vue par ses auteurs," (propos recueillis par Alain Garel), Image et son, 316, 1977, p.23.

4 Cela contredit la conclusion de Gian Piero Brunetta qui dans son introduction à Arrivano $i$ Mostri: $i$ volti della commedia italiana de Jean A. Gili, (Bologna: Cappelli, 1980), p. 7 à 28 , déclare que "la comédie en tant que genre est en pleine crise."

5 Alain Garel, "La comédie italienne et la critique," Image et son, 315, 1977, p.30. 
6 Robert Benayoun, "L'Italien millionnaire" in Positif 23 (avril 57), 18.

7 Aldo Tassone l'affirme dans un article intitulé "Du néo-réalisme aux années soixante," Cinéma 74 (1974), 190-191.

8 Lino Miccichè, "Linee e tendenze del cinema italiano," Cinema 81 (Milano: Feltrinelli, 1981), p. 11.

9 L'année dernière, à l'occasion du Cinquantenaire de la Mostra biennale de Venise, Gassman présentait son dernier film "Di padre in figlio." "Nudo di donna" est le dernier long métrage de Nino Manfredi; "lo so che tu sai che io so," l'oeuvre la plus récente de Sordi et "I viaggiatori della sera" de Tognazzi date de 1979.

10 Op.cit., p.18.

11 A ce sujet, lire l'introduction de Gian Piero Brunetta, "I Mostri et gli altri animali" dans Arrivano i Mostri, op.cit., pp. 7-28. En même temps, la comédie souligne, sous un angle critique, l'éclatement des institutions: famille, magistrature, église et police. Cf. les filmographies aux pp.: 57 à 60, 87 à 90, 121 à 125 et 145 à 149.

12 Scénariste, comédien, metteur en scène comme les autres auteurs comiques de sa génération, Massimo Troisi, âgé de vingt neuf ans, est un méridional né à San Giorgio a Cremano, non loin de Naples. Bien qu'issu d'une famille pauvre, il poursuit ses études et obtient un diplôme de géomètre, avant de se consacrer au théâtre, puisant dans le répertoire sérieux de Eduardo, Petito et Viviani. En 1976, il fonde avec Lello Arena et Enzo Decaro, la Smorfia, un trio dont les sketches deviendront célèbres par l'entremise de la télévision. En 1979, le groupe se dissout et, peu de temps après, Massimo Troisi se lance dans le cinéma. Son premier film "Ricomincio da tre," bien que difficilement compris par les septentrionaux qui ne connaissent pas le dialecte napolitain, pulvérise en 1981 tous les records d'entrée en Italie, car on y décèle les brillantes prémices d'une nouvelle forme de comédie.

13 Dans les films de Nichetti, comme dans ceux de Tati, tout est à voir au sens premier de l'image et les rares ingérences du dialogue dans l'action provoquent des effets de trouble chez le héros. 